\title{
Uncovering Quercetin's Effects against Influenza A Virus Using Network Pharmacology and Molecular Docking
}

\author{
Minjee Kim and Young Bong Kim *
}

check for updates

Citation: Kim, M.; Kim, Y.B. Uncovering Quercetin's Effects against Influenza A Virus Using Network Pharmacology and Molecular Docking. Processes 2021, 9 , 1627. https://doi.org/10.3390/ pr9091627

Academic Editor: Jing Tang

Received: 3 July 2021

Accepted: 7 September 2021

Published: 9 September 2021

Publisher's Note: MDPI stays neutral with regard to jurisdictional claims in published maps and institutional affiliations.

Copyright: (C) 2021 by the authors Licensee MDPI, Basel, Switzerland. This article is an open access article distributed under the terms and conditions of the Creative Commons Attribution (CC BY) license (https:/ / creativecommons.org/licenses/by/ $4.0 /)$.
Department of Biomedical Science and Engineering, Konkuk University, Seoul 05029, Korea; mj0411@konkuk.ac.kr

* Correspondence: kimera@konkuk.ac.kr; Tel.: +82-2-450-4208

\begin{abstract}
Background: Re-emerging influenza threats continue to challenge medical and public health systems. Quercetin is a ubiquitous flavonoid found in food and is recognized to possess antioxidant, anti-inflammatory, antiviral, and anticancer activities. (2) Methods: To elucidate the targets and mechanisms underlying the action of quercetin as a therapeutic agent for influenza, network pharmacology and molecular docking were employed. Biological targets of quercetin and target genes associated with influenza were retrieved from public databases. Compound-disease target (C-D) networks were constructed, and targets were further analyzed using KEGG pathway analysis. Potent target genes were retrieved from the compound-disease-pathway (C-D-P) and protein-protein interaction (PPI) networks. The binding affinities between quercetin and the targets were identified using molecular docking. (3) Results: The pathway study revealed that quercetin-associated influenza targets were mainly involved in viral diseases, inflammation-associated pathways, and cancer. Four targets, MAPK1, NFKB1, RELA, and TP53, were identified to be involved in the inhibitory effects of quercetin on influenza. Using the molecular docking method, we evaluated the binding affinity of each ligand (quercetin)-target and discovered that quercetin and MAPK1 showed the strongest calculated binding energy among the four ligand-target complexes. (4) Conclusion: These findings identified potential targets of quercetin and suggest quercetin as a potential drug for influenza treatment.
\end{abstract}

Keywords: quercetin; influenza; network pharmacology; molecular docking; systems biology

\section{Introduction}

Seasonal influenza A virus (IAV) is an infectious, enveloped, negative-sense singlestranded RNA virus that is the most common cause of pneumonia-related deaths [1]. Symptoms of IAV infection may range from a mild respiratory disease, characterized by fever, cough, muscle pain, and fatigue, to severe lethal pneumonia due to secondary bacterial infection [2]. Vaccines are administered as a prophylactic treatment but must be reformulated every year due to the antigenic drift of the influenza virus [2]. In the case of anti-influenza therapy, only a few drugs have been approved for clinical use, one targeting the influenza M2 ion channel protein, and the other targeting neuraminidase (NA) [3]. However, the corresponding resistance against current drugs still poses a challenge for the treatment of influenza. Therefore, the current treatment goal for influenza is to develop a universal therapy that is easily accessible and provides protection against influenza to combat pandemics.

Due to the emerging mutation of the influenza virus and drug resistance, many patients turn to complementary and alternative therapies. With regard to antiviral drugs, 37\% of the 98 entities registered from 1981 to 2019 were derived from natural product botanical medicines [4]. Natural drugs are important alternative therapies for the treatment of influenza, and many reports have shown that the development of novel bioactive chemicals extracted from natural drugs has significant advantages [5]. Oseltamivir is an example of a successful anti-influenza drug synthesized using two natural products, quinic acid 
and shikimic acid, as starting materials [6]. Phytochemicals such as flavonoids are widely consumed in our daily foods, and previous studies have reported the protective effects of flavonoids against influenza, inflammation, and other viral infections [7]. Quercetin is one of the most common flavonoids that exerts antioxidative, antibacterial, and antiviral effects [8]. A recent study indicated that quercetin showed inhibitory activity in the early stage of influenza infection via interaction with viral hemagglutinin (HA) protein [8]. However, the therapeutic targets and molecular mechanisms underlying the action of quercetin against influenza have not been fully studied.

Due to complex matrices of natural products, elucidation of the molecular mechanisms underlying the action of natural product-based drugs is difficult owing to the synergistic effects of the active compounds and multiple therapeutic targets involved. With the rapid progress of systems biology, network-based drug discovery is considered a promising approach towards cost-effective and time-efficient drug development. Network pharmacology is an emerging approach that highlights the concept of "network targets and multicomponent therapeutics" [9]. Herein, we aimed to identify the targets associated with quercetin and influenza to determine common targets. Common targets were further studied through the construction of a protein-protein interaction (PPI) network. We confirmed biological signaling pathways based on common targets and constructed a compound-disease-target-biological signaling pathway (C-D-P) network to understand the mechanisms underlying the interactions between quercetin and influenza. Ligandtarget molecular docking was conducted to identify the most potent target. In this study, we aimed to determine the potent targets and mechanism underlying the action of quercetin against influenza.

\section{Materials and Methods}

\subsection{Pharmacokinetic Evaluation and Therapeutic Targets Associated with Quercetin}

The therapeutic targets of quercetin were obtained from the DrugBank (ver. 5.1.8), GeneCards (ver. 5.0), and NCBI databases. DrugBank is a unique bioinformatics resource that combines detailed drug with comprehensive drug target (i.e., protein) information [10]. The database contains 14,556 drug entries, including 2699 approved small molecule drugs, 6653 experimental drugs, and 5259 non-redundant proteins (i.e., drug targets) [10]. GeneCards is an integrative and automatically mined database that provides genomic, transcriptomic, proteomic, genetic, clinical, and functional information. GeneCards uses 176 sources and external links to implement drug targets [11]. The NCBI gene database integrates information from a wide range of species and supplies genespecific connections in sequence, expression, structure, and function [12]. All searches performed were specific to Homo sapiens.

\subsection{Potential Pathological Target Genes Linked to Influenza}

Influenza-associated targets were obtained from the DisGeNET (ver. 6.0) and NCBI databases. DisGeNET integrates information on human gene-disease associations and variant-disease associations from various resources including Mendelian, complex, and environmental diseases performed by gene and disease vocabulary mapping [13]. All searches performed were specific to Homo sapiens.

\subsection{Construction of Compound-Disease Target (C-D) Network}

Overlapping genes between quercetin (compound) and influenza (disease) targets were retrieved and constructed as a network using Cytoscape (ver. 3.8) software.

\subsection{Biological Function and Pathway Enrichment Analysis}

Pathways associated with the target proteins were retrieved from the Database for Annotation, Visualization and Integrated Discovery (DAVID, ver. 6.8). Kyoto Encyclopedia of Genes and Genomes (KEGG) pathway enrichment analysis was conducted through DAVID. DAVID bioinformatics is an analytic tool aimed to extract biological meaning 
from large gene and protein lists. Based on the selected gene list, the database uses one or more text- and pathway-mining tools such as gene functional classification, functional annotation charts, or clustering and functional annotation tables. This method uses statistical approaches to identify significantly enriched or depleted groups of selected genes or proteins [14].

\subsection{Compound-Disease Target-Pathway (C-D-P) Network Construction}

Compound-disease target and disease target-pathway networks were constructed and visualized using Cytoscape. Pathways with $p<0.05$ were used in this network. Two constructed networks were analyzed and merged to produce a compound-disease target-pathway (C-D-P) network.

\subsection{Construction of Protein-Protein Interaction (PPI) Network}

The STRING database (ver. 11.0) was used to obtain target-target interactions, with a confidence score $>0.7$. STRING is a database of known and predicted protein-protein interactions that currently covers $24,584,628$ proteins from 5090 organisms. The interactions include direct (physical) and indirect (functional) associations. Based on uploaded gene sets, the database visualizes interaction networks and performs gene-set enrichment analysis on the entire input [15].

\subsection{Molecular Docking Analysis}

A ligand-target docking approach was used to analyze structural complexes of the target with ligands to understand target specificity. The three-dimensional (3D) structure of the target was downloaded from the RCSB protein data bank. Quercetin was obtained from the PubChem database and converted to PDB files using the Avogadro program (ver. 1.90.0). Docking was carried out by AutoDock Vina (ver. 1.1.2) using PyRx (ver. 0.9.6) based on scoring functions. The 2D and 3D ligand-target interactions were retrieved using BIOVIA Discovery Studio (ver. 4.5).

\section{Results}

3.1. Biological Targets of Quercetin and Influenza and Compound-Disease Target Network Construction (C-D Network)

Overall, 130 target genes (H. sapiens) associated with quercetin were retrieved from the DrugBank and NCBI databases. A total of 838 targets associated with influenza were identified from the DisGeNET and NCBI databases (Figure 1A). Between 130 quercetin targets and 838 influenza targets, we retrieved 30 overlapping target genes as influenzaassociated quercetin targets. Based on 30 genes, we constructed a compound (quercetin)disease (influenza infection) (C-D) network using Cytoscape.

\subsection{KEGG Pathway Analysis}

Thirty targets retrieved from the database were further analyzed using DAVID bioinformatics resources for KEGG enrichment analysis. Table 1 lists the top 15 enriched pathways and diseases $(p<0.05)$ based on gene counts. Specifically, these targets were enriched with six viral diseases (hepatitis $\mathrm{B}$, tuberculosis, Chagas disease, toxoplasmosis, hepatitis $\mathrm{C}$, and influenza A), three inflammation-associated pathways (TNF, MAPK, and PI3K-AKT signaling pathways), and cancer (Table 1). 
(A)

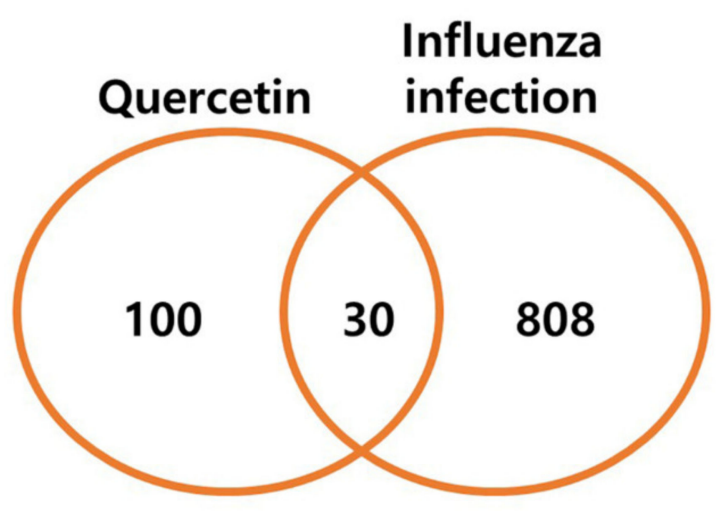

(B)

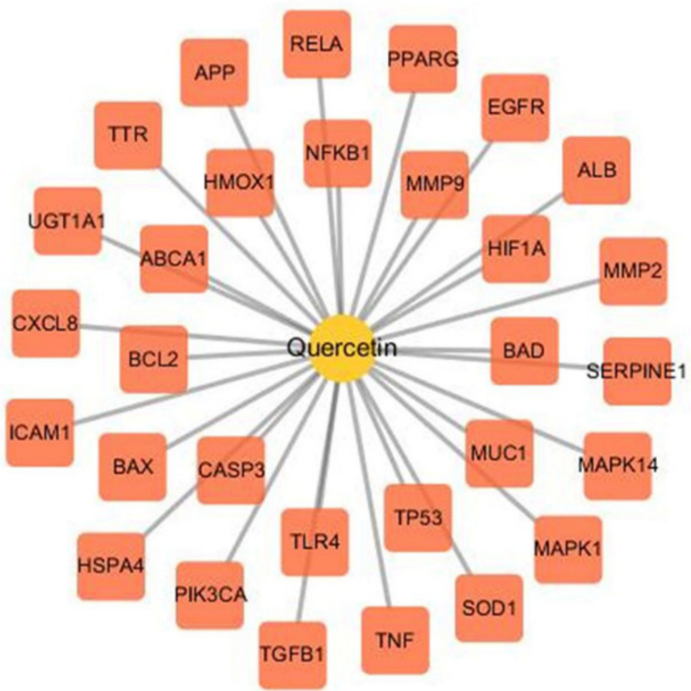

Figure 1. (A) Biological targets of quercetin and influenza infection. Thirty overlapping targets were identified. (B) Quercetin (compound)-Influenza infection (disease) target network (C-D network).

Table 1. Pathway and Disease KEGG pathway.

\begin{tabular}{cc}
\hline Pathway and Disease (KEGG) & Gene Counts \\
hsa05200: Pathways in cancer & 16 \\
hsa05161: Hepatitis B & 14 \\
hsa05205: Proteoglycans in cancer & 12 \\
hsa05152: Tuberculosis & 11 \\
hsa04066: HIF-1 signaling pathway & 10 \\
hsa05142: Chagas disease (American trypanosomiasis) & 10 \\
hsa05145: Toxoplasmosis & 10 \\
hsa05160: Hepatitis C & 10 \\
hsa04210: Apoptosis & 9 \\
hsa04668: TNF signaling pathway & 9 \\
hsa04722: Neurotrophin signaling pathway & 9 \\
hsa04071: Sphingolipid signaling pathway & 9 \\
hsa05164: Influenza A & 9 \\
hsa04010: MAPK signaling pathway & 9 \\
hsa04151: PI3K-Akt signaling pathway & 9 \\
\hline
\end{tabular}

\subsection{Compound-Disease-Target-Pathway (C-D-P) Network}

The disease-target-pathway network (D-P) was constructed and merged with the C-D network (Figure 1A) to yield the C-D-P network (Figure 2). Our C-D-P network yielded 46 nodes and 186 edges. Within the network, the nodes represent different targets and the edges indicate intermolecular interactions between nodes [16]. The network was analyzed by using the Cytoscape plugin 'NetworkAnalyzer' [17]. Among the target nodes, MAPK1, NFKB1, and RELA showed the highest degree of 14. Degree indicates the number of edges linked to a given node. Therefore, nodes with a high degree may represent the hub genes possessing important biological functions [17]. The size of target nodes (dark orange) corresponds to the number of degrees. In short, we considered nodes carrying high degrees to be the hub genes. 


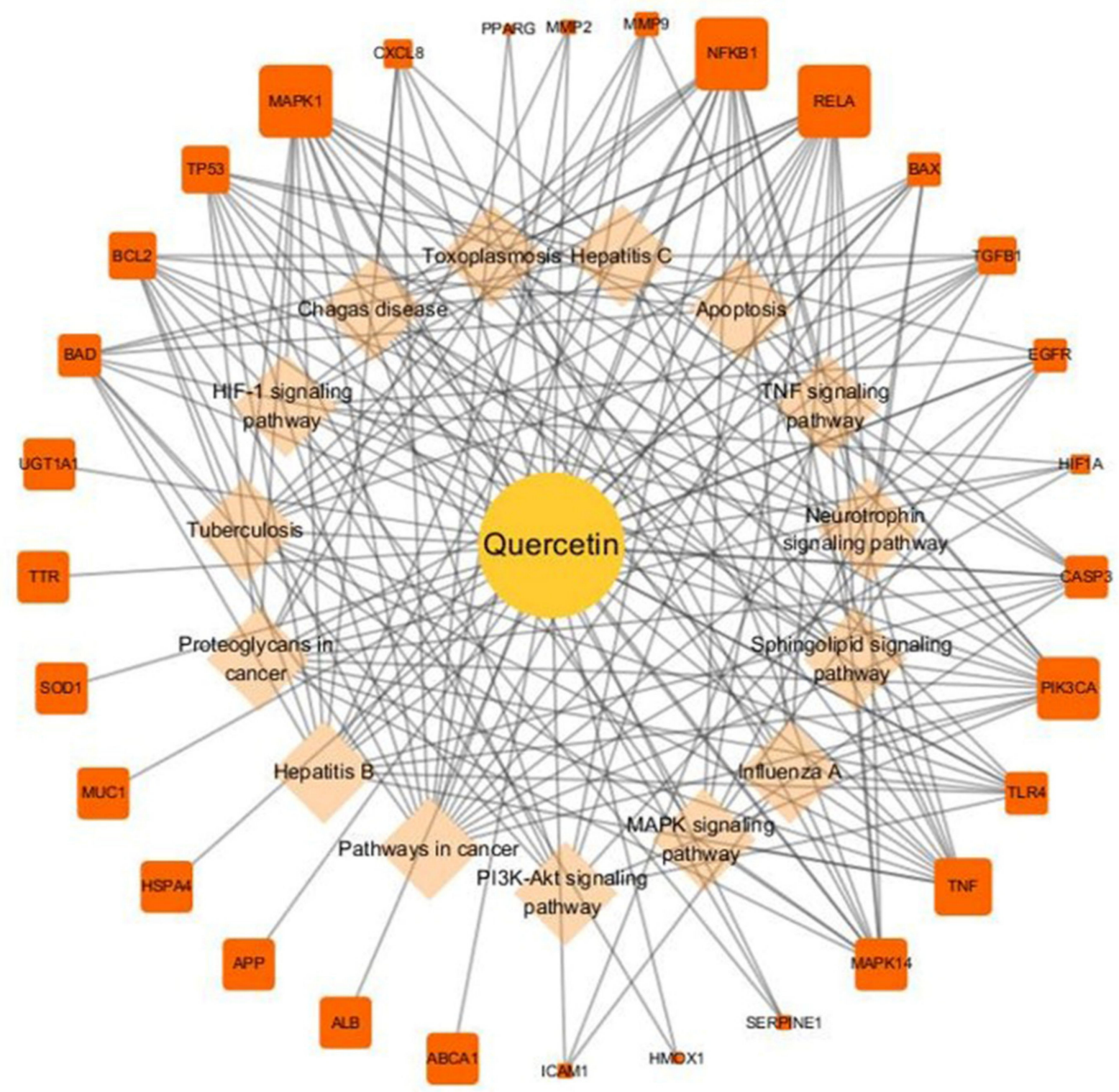

Figure 2. Compound-disease-target-pathway (C-D-P) network. The network has 46 nodes and 186 edges. Among the target nodes, MAPK1, NFKB1, and RELA showed the highest degree of 14. The size of target nodes (dark orange) corresponds to the number of degrees.

\subsection{Protein-Protein Interaction of Disease Targets Associated with Quercetin}

From the STRING database, 30 target genes were inputted to retrieve a protein-protein interaction (PPI) network (Figure 3). The network was analyzed based on the size of the degree using Cytoscape. We identified two main targets: TP53, which showed the highest degree of 20, followed by MAPK1 (Degree 19). 


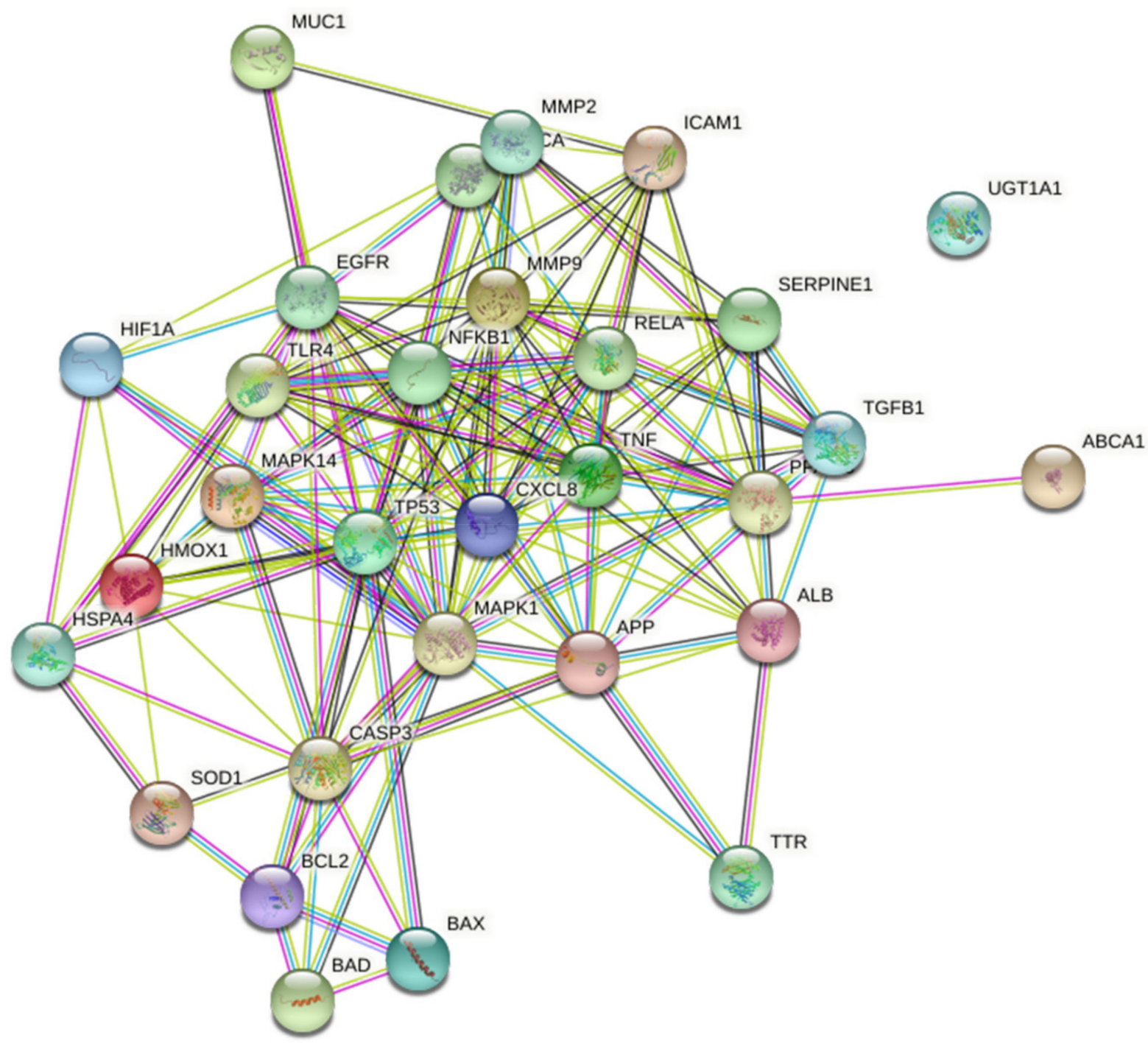

Figure 3. Protein-Protein Interaction (PPIs) network using STRING database. Two main targets, TP53, which showed the highest degree of 20, followed by MAPK1 (Degree 19) were identified.

\subsection{Molecular Docking Analysis}

From our two network results (Figures 2 and 3), we identified MAPK1, NFKB1, RELA, and TP53 as four potential targets for quercetin against influenza. A ligand-target docking approach was used to analyze structural complexes of targets: MAPK1 (PDB ID: 4H3P); NFKB1 (PDB ID: 3GUT); RELA (PDB ID: 1NFI); TP53: (PDB ID: 5Z78) with ligand (Quercetin, QT) using PyRx virtual screening tool (Table 2).

Table 2. Ligand-Target binding affinity.

\begin{tabular}{cc}
\hline Ligand-Target & Binding Affinity (kcal/moL) \\
\hline QT-MAPK1 & -8.3 \\
\hline QT-NFKB1 & -7.3 \\
\hline QT-RELA & -7.1 \\
\hline QT-TP53 & -6.1 \\
\hline
\end{tabular}


We retrieved the binding affinity of each ligand-target complex and discovered that quercetin and MAPK1 showed the strongest binding affinity of $-8.3 \mathrm{kcal} / \mathrm{mol}$ among the four complexes.

The ligand-receptor interaction was identified using the Discovery Studio Visualizer (Figure 4). The 3D- and 2D-binding models of quercetin-MAPK1 was further analyzed. Figure $4 \mathrm{~A}$ shows the full image of quercetin-MAPK1 interaction in a 3D representation. Figure 4B shows the interaction site of MAPK1 with quercetin. Key residues were analytically labeled and visualized in a 2D model to understand the details of the binding of the target to quercetin (Figure 4C). The compound quercetin was found to interact with MAPK1 amino acids VAL173 and LYS 330 via $\pi$-alkyl bonds and HIS 61 via hydrogen bonds and $\pi$-cation bonds. \%clearpage

(A)

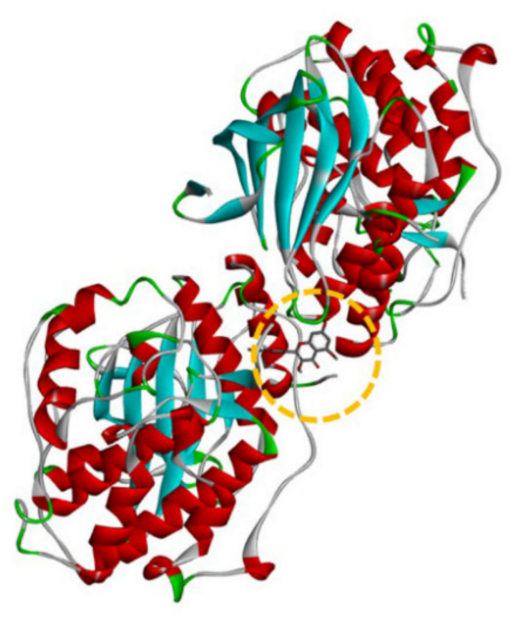

(B)

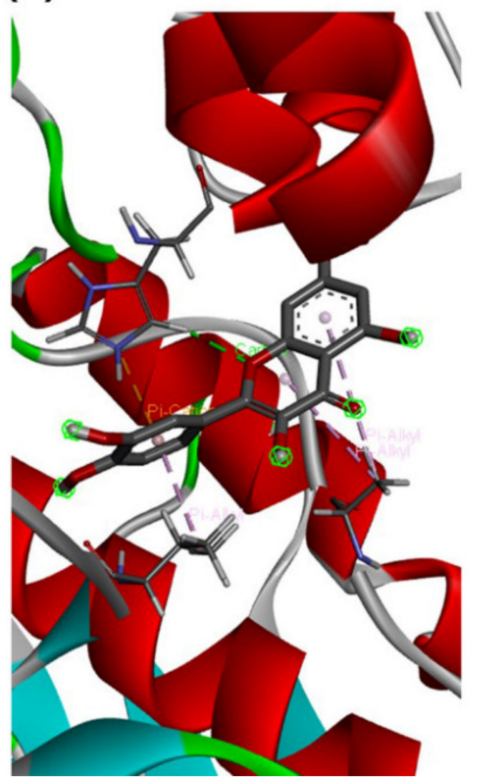

(C)

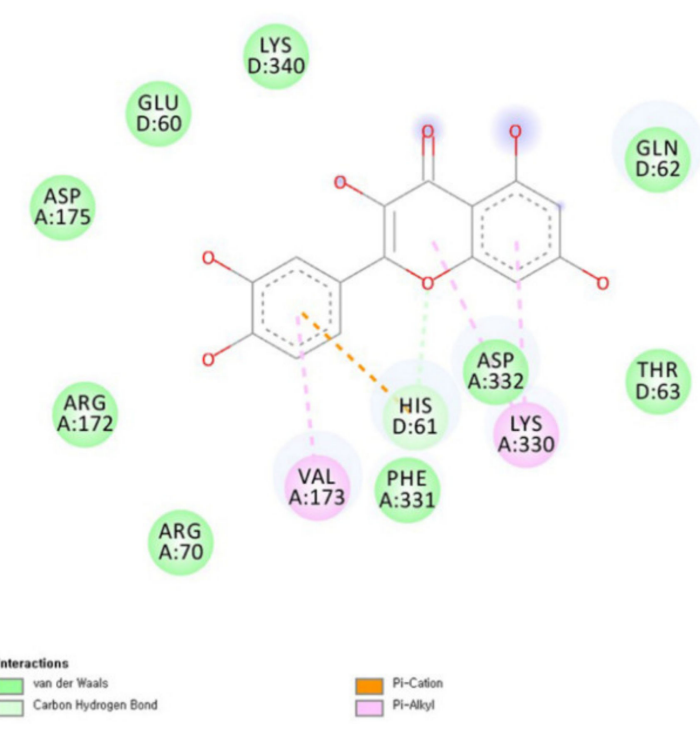

Figure 4. Ligand-receptor binding simulation of quercetin and target (MAPK1) using Discovery Studio Visualizer. (A) The 3D representation of quercetin-MAPK1 interaction. (B) 3D representation of quercetin bound to MAPK1 receptor site.

(C) The 2Drepresentation of quercetin-MAPK interaction tagged with key residues.

\section{Discussion}

Given the rapid and advanced progress in systems biology, network pharmacology is considered to be the next paradigm in cost-effective drug development that can provide molecular-level insights into pharmacological studies $[9,18]$. In this research, a systems biology approach that integrated target identification, network construction, KEGG pathway enrichment, and molecular docking was established to systematically analyze the potential target of quercetin against influenza. From our database search, we observed 30 quercetin targets associated with influenza and confirmed that the targets were mainly associated with pathways such as viral diseases (hepatitis B, tuberculosis, Chagas disease, toxoplasmosis, hepatitis $\mathrm{C}$, and influenza A), inflammation-associated pathways (TNF, MAPK, and PI3K-AKT signaling pathways), and cancer. Network analysis revealed four main targets among the 30 targets, MAPK1, NFKB1, RELA, and TP53, which were identified to be most potent. The molecular docking method was implemented to evaluate the binding affinities between quercetin and the selected targets. We observed that quercetin and MAPK1 showed the strongest binding affinity, indicating that MAPK1 can be a novel target associated with influenza.

IAV continues to emerge annually, resulting in significant morbidity and mortality, and poses a serious threat to public health and socio-economic factors [19]. Due to the emergence of antigenic drift and subsequent drug-resistance, the clinical use of IAV vaccines 
is associated with limitations. Many reports of MAPK signaling induced by negativestrand RNA viruses are now accumulating. The MAPK family is subdivided into three subunits: extracellular signal-regulated kinase (ERK), p38 MAPK, and c-Jun-NH2-terminal kinase (JNK) [20]. Airway epithelial cells are the initial target of IAV, and produce various inflammatory mediators including prostaglandin. The MAPK family is activated by IAV and induces prostaglandin E2 (PGE2) synthesis from arachidonic acid in bronchial epithelial cells leading to respiratory inflammation [20]. ERK has also been reported to be involved in vacuolar $\mathrm{H}^{+}$-ATPase activity to acidify endosomes and affect the fusion of the viral and endosomal membranes during the early stage of IAV infection [21]. Previous studies reported that quercetin reduced activation of phosphorylated ERK kinase and p38 MAP kinase but not JNK MAP kinase in lipopolysaccharide (LPS)-stimulated macrophage [22]. In addition, quercetin treatment inhibited NF- $\mathrm{KB}$ activation (associated with our target search: NFKB1, RELA) and proinflammatory cytokines, which corresponds to our target result [22]. Therefore, based on previous papers, quercetin may inhibit MAPK and NF-kB activation induced by influenza infection. Another research group reported quercetin's inhibitory effect on TNF- $\alpha$-stimulated PI3-kinase activity and Akt phosphorylation, which supports our KEGG pathway study result [23]. PI3-kinase and Akt have been shown to be required for the maximal activation of NF- $\mathrm{kB}$ in response to TNF- $\alpha$ and other stimuli. Quercetin inhibited TNF- $\alpha$-induced chemokine expression by attenuating NF-KB transactivation via a PI3-kinase/Akt dependent pathway, which in lines with our pathway and target results [23]. Wu et al. observed that the inhibitory effect of quercetin against IAV was enhanced when IAV was pre-incubated or co-incubated with quercetin, suggesting that quercetin may effectively inhibit viral infection during the viral entry stage [8]. Therefore, quercetin can be a promising candidate as a rational drug for IAV treatment.

In conclusion, our study may act as a guideline to the molecular targets and underlying mechanisms of quercetin in treating influenza infection and applies an in silico approach to drug development. Big-data-driven public databases have been evolving with ever-expanding data and are considered key assets in drug development [24,25]. However, these databases are often limited by the up-to-date knowledge harvested by published studies [24]. Computational-based approaches may be difficult to use to find a novel drug that can be realized by experimental studies, but it could be an attractive new solution to drug repositioning with the concept of "network targets and multicomponent therapeutics" [9]. Moreover, in silico studies may bring impacts on clinical research by reducing animal studies and human cohorts, enabling precision medicine for complex diseases [25]. The biological and clinical data in large scale have enabled computational drug development more relevant to the real world, particularly in identification of therapeutic targets for specific diseases [25]. Although it is imperative that our selected targets be tested clinically, which is one of the limitations of our study. This systems biology study may contribute to a better understanding of the mechanisms underlying the action of quercetin against influenza infection.

Author Contributions: Conceptualization, M.K.; methodology, M.K.; software, M.K.; validation, M.K.; data curation, M.K.; writing—original draft preparation, M.K.; writing-review and editing, Y.B.K.; visualization, M.K.; supervision, Y.B.K.; funding acquisition, Y.B.K. All authors have read and agreed to the published version of the manuscript.

Funding: This research was supported by a grant of the Korea Health Technology R\&D Project through the Korea Health Industry Development Institute (KHIDI), funded by the Ministry of Health \& Welfare (grant No. HI15C2842, HI15C1685, HI18C2177).

Informed Consent Statement: Not applicable.

Conflicts of Interest: The authors declare no conflict of interest. 


\section{References}

1. Herold, S.; Becker, C.; Ridge, K.M.; Budinger, G.R. Influenza virus-induced lung injury: Pathogenesis and implications for treatment. Eur. Respir. J. 2015, 45, 1463-1478. [CrossRef]

2. Krammer, F.; Smith, G.J.D.; Fouchier, R.A.M.; Peiris, M.; Kedzierska, K.; Doherty, P.C.; Palese, P.; Shaw, M.L.; Treanor, J.; Webster, R.G.; et al. Influenza. Nat. Rev. Dis. Primers 2018, 4, 3. [CrossRef]

3. Wu, X.; Sun, Q.; Zhang, C.; Yang, S.; Li, L.; Jia, Z. Progress of small molecular inhibitors in the development of anti-influenza virus agents. Theranostics 2017, 7, 826-845. [CrossRef]

4. Newman, D.J.; Cragg, G.M. Natural Products as Sources of New Drugs over the Nearly Four Decades from 01/1981 to 09/2019. J. Nat. Prod. 2020, 83, 770-803. [CrossRef] [PubMed]

5. Zhang, Z.J.; Morris-Natschke, S.L.; Cheng, Y.Y.; Lee, K.H.; Li, R.T. Development of anti-influenza agents from natural products. Med. Res. Rev. 2020, 40, 2290-2338. [CrossRef]

6. Kim, C.U.; Lew, W.; Williams, M.A.; Liu, H.; Zhang, L.; Swaminathan, S.; Bischofberger, N.; Chen, M.S.; Mendel, D.B.; Tai, C.Y.; et al. Influenza neuraminidase inhibitors possessing a novel hydrophobic interaction in the enzyme active site: Design, synthesis, and structural analysis of carbocyclic sialic acid analogues with potent anti-influenza activity. J. Am. Chem. Soc. 1997, 119, 681-690. [CrossRef]

7. Choi, H.J.; Song, J.H.; Park, K.S.; Kwon, D.H. Inhibitory effects of quercetin 3-rhamnoside on influenza A virus replication. Eur. J. Pharm. Sci. Off. J. Eur. Fed. Pharm. Sci. 2009, 37, 329-333. [CrossRef]

8. Wu, W.; Li, R.; Li, X.; He, J.; Jiang, S.; Liu, S.; Yang, J. Quercetin as an Antiviral Agent Inhibits Influenza A Virus (IAV) Entry. Viruses 2015, 8, 6. [CrossRef] [PubMed]

9. Hopkins, A.L. Network pharmacology: The next paradigm in drug discovery. Nat. Chem. Biol. 2008, 4, 682-690. [CrossRef] [PubMed]

10. Wishart, D.S.; Knox, C.; Guo, A.C.; Shrivastava, S.; Hassanali, M.; Stothard, P.; Chang, Z.; Woolsey, J. DrugBank: A comprehensive resource for in silico drug discovery and exploration. Nucleic Acids Res. 2006, 34, D668-D672. [CrossRef] [PubMed]

11. Stelzer, G.; Rosen, N.; Plaschkes, I.; Zimmerman, S.; Twik, M.; Fishilevich, S.; Stein, T.I.; Nudel, R.; Lieder, I.; Mazor, Y.; et al. The GeneCards Suite: From Gene Data Mining to Disease Genome Sequence Analyses. Curr. Protoc. Bioinform. 2016, 54, 1-30. [CrossRef]

12. NCBI Resource Coordinators. Database resources of the National Center for Biotechnology Information. Nucleic Acids Res. 2018, 46, D8-D13. [CrossRef] [PubMed]

13. Piñero, J.; Ramírez-Anguita, J.M.; Saüch-Pitarch, J.; Ronzano, F.; Centeno, E.; Sanz, F.; Furlong, L.I. The DisGeNET knowledge platform for disease genomics: 2019 update. Nucleic Acids Res. 2019, 48, D845-D855. [CrossRef] [PubMed]

14. Huang, W.; Sherman, B.T.; Lempicki, R.A. Systematic and integrative analysis of large gene lists using DAVID bioinformatics resources. Nat. Protoc. 2009, 4, 44-57. [CrossRef]

15. Szklarczyk, D.; Gable, A.L.; Lyon, D.; Junge, A.; Wyder, S.; Huerta-Cepas, J.; Simonovic, M.; Doncheva, N.T.; Morris, J.H.; Bork, P.; et al. STRING v11: Protein-protein association networks with increased coverage, supporting functional discovery in genome-wide experimental datasets. Nucleic Acids Res. 2019, 47, D607-D613. [CrossRef]

16. Shannon, P.; Markiel, A.; Ozier, O.; Baliga, N.S.; Wang, J.T.; Ramage, D.; Amin, N.; Schwikowski, B.; Ideker, T. Cytoscape: A software environment for integrated models of biomolecular interaction networks. Genome Res. 2003, 13, 2498-2504. [CrossRef]

17. Assenov, Y.; Ramírez, F.; Schelhorn, S.-E.; Lengauer, T.; Albrecht, M. Computing topological parameters of biological networks. Bioinformatics 2007, 24, 282-284. [CrossRef]

18. Zhang, R.; Zhu, X.; Bai, H.; Ning, K. Network Pharmacology Databases for Traditional Chinese Medicine: Review and Assessment. Front. Pharmacol. 2019, 10, 123. [CrossRef]

19. Yang, J.; Li, M.; Shen, X.; Liu, S. Influenza A virus entry inhibitors targeting the hemagglutinin. Viruses 2013, 5, 352-373. [CrossRef]

20. Mizumura, K.; Hashimoto, S.; Maruoka, S.; Gon, Y.; Kitamura, N.; Matsumoto, K.; Hayashi, S.; Shimizu, K.; Horie, T. Role of mitogen-activated protein kinases in influenza virus induction of prostaglandin E2 from arachidonic acid in bronchial epithelial cells. Clin. Exp. Allergy J. Br. Soc. Allergy Clin. Immunol. 2003, 33, 1244-1251. [CrossRef] [PubMed]

21. Marjuki, H.; Alam, M.I.; Ehrhardt, C.; Wagner, R.; Planz, O.; Klenk, H.D.; Ludwig, S.; Pleschka, S. Membrane accumulation of influenza A virus hemagglutinin triggers nuclear export of the viral genome via protein kinase Calpha-mediated activation of ERK signaling. J. Biol. Chem. 2006, 281, 16707-16715. [CrossRef]

22. Cho, S.Y.; Park, S.J.; Kwon, M.J.; Jeong, T.S.; Bok, S.H.; Choi, W.Y.; Jeong, W.I.; Ryu, S.Y.; Do, S.H.; Lee, C.S.; et al. Quercetin suppresses proinflammatory cytokines production through MAP kinases andNF-kappaB pathway in lipopolysaccharide-stimulated macrophage. Mol. Cell Biochem. 2003, 243, 153-160. [CrossRef]

23. Nanua, S.; Zick, S.M.; Andrade, J.E.; Sajjan, U.S.; Burgess, J.R.; Lukacs, N.W.; Hershenson, M.B. Quercetin blocks airway epithelial cell chemokine expression. Am. J. Respir. Cell Mol. Biol. 2006, 35, 602-610. [CrossRef] [PubMed]

24. Casciuc, I.; Horvath, D.; Gryniukova, A.; Tolmachova, K.A.; Vasylchenko, O.V.; Borysko, P.; Moroz, Y.S.; Bajorath, J.; Varnek, A. Pros and cons of virtual screening based on public "Big Data": In silico mining for new bromodomain inhibitors. Eur. J. Med. Chem. 2019, 165, 258-272. [CrossRef] [PubMed]

25. Qian, T.; Zhu, S.; Hoshida, Y. Use of big data in drug development for precision medicine: An update. Expert Rev. Precis. Med. Drug Dev. 2019, 4, 189-200. [CrossRef] 